\title{
Dust production and coma morphology of 67P/Churyumov-Gerasimenko during the 2002-2003 apparition
}

\author{
M. R. Kidger ${ }^{\star}$ \\ Instituto de Astrofísica de Canarias (IAC), C/Vía Láctea s/n, 38205 La Laguna, Tenerife, Spain
}

Received 27 May 2003 / Accepted 17 June 2003

\begin{abstract}
Light curves and images of 67P/Churyumov-Gerasimenko are presented for the 2002-2003 apparition based on amateur CCD monitoring using a standardised method to produce consistent photometry in Johnson-Kron-Cousins system. Multiaperture data shows a large outburst at perihelion during which dust production increased by a factor of 2 to give a peak value of $A f \rho \approx 350 \mathrm{~cm}$. A similar outburst was seen at the same epoch of the previous apparition in 1995-96 and probably in 1982-83, suggesting that the light curve is similar at succesive returns. A considerable change is seen in the slope of the light curve at $r=1.85 \mathrm{AU}(T+140$ days) corresponding to a second significant increase in dust production. Overall though the dust production shows a very steep dependence with heliocentric distance, falling as $r^{-5.8}$.
\end{abstract}

Key words. comets: individual: 67P/Churyumov-Gerasimenko - techniques: photometric

\section{Introduction}

Comet 67P/Churyumov-Gerasimenko is the new target of the ROSETTA mission after its failure to meet the strict launch window for an Ariane-boosted encounter with the original target 46P/Wirtanen. The comet was discovered on 1969 Sep. 11 on plates taken for astrometry of 32P/Comas-Solá. The comet has been seen at 6 apparitions, of which the latest was in 2002-2003, with perihelion on 2002 Aug. 18. The perihelion distance is $1.29 \mathrm{AU}$ and the period $6.57 \mathrm{yrs}$; both values are slightly larger than the corresponding ones for $46 \mathrm{P} /$ Wirtanen of $1.06 \mathrm{AU}$ and 5.44 yrs.

The dynamic history of $67 \mathrm{P} /$ Churyumov-Gerasimenko shows two significant drops in perihelion distance in the last 160 yrs. The orbit was initially investigated by Beliaev (1974), who found Jupiter encounters in 1840 and 1959 that reduced the perihelion distance significantly. However Beliaev et al. (1986) and Carusi et al. (1985) found that the importance of the 1840 Jupiter encounter had been overestimated in the initial study. A further encounter with Jupiter in 1959 reduced the perihelion distance from $q=2.75$ AU to the present value. The perihelion distance has remained stable since discovery. Comets that show such drops in perihelion distance in their recent dynamical history are generally more active than objects that have had a stable orbit and often show activity even at aphelion. It is believed that the drop in perihelion distance removes the surface dust mantle that acts as thermal insulation, suffocating the sublimation of volatiles. When the perihelion distance drops, additional gas pressure from sublimation builds

\footnotetext{
^ e-mail: mrk@ll.iac.es
}

up under the mantle leading to its expulsion. The comet will then show relatively high activity until a new mantle is built up over the course of a number of returns to perihelion.

Tancredi et al. (2000) estimate an absolute magnitude of the nucleus of $H_{0}=15.6$ and hence a radius of $2.5 \mathrm{~km}$ for 67P/Churyumov-Gerasimenko, making it one of the larger comets in the Jupiter family. This makes it a significantly larger object than the initial target for which the ROSETTA mission was designed (46P/Wirtanen has $H_{0}=18.4$ and an estimated radius of $0.7 \mathrm{~km}$ ). Both radii are rated to have Quality 1 and thus have small errors. Comparison with HST data for comets in common between the Tancredi (2000) data and the HST data set shows good agreement between estimates of nuclear radius despite the radically different methods used. Radar observations in 1982 by Kamoun et al. (1998) show a $3 \sigma$ upper limitdetection that was considered "too weak to claim a detection", but that gave a hard upper limit of $3.7 \mathrm{~km}$ for the radius of the nucleus and a probable upper limit of $3 \mathrm{~km}$, consistent with the estimate of Tancredi et al. (2000). Similarly, Mueller (1992) estimates a radius of $3.2 \mathrm{~km}$ with an albedo of 0.03 and minimum $a / b$ axis ratio of 1.7 , in good agreement with the previously cited values.

Due to the important differences, particularly in radius and activity between 46P/Wirtanen and 67P/ChuryumovGerasimenko careful characterisation of the comet is important to the success of the ROSETTA mission. 67P/ChuryumovGerasimenko is considered to be a dusty comet (Kiselev 1998), putting it in a group that includes such objects as $1 \mathrm{P} /$ Halley, 4P/Faye, 21P/Giacobini-Zinner, and 22P/Kopff, as well as nonperiodic objects such as C/1996 B2 (Hyakutake), C/1995 O1 
(Hale-Bopp) and C/1975 V1 (West). In this paper we study the light curve and derive the dust activity using the parameter $A f \rho$.

\section{Observations}

The widespread use of CCDs by amateur astronomers has led to a huge increase in the amount of astrometry reported to the Minor Planet Center. Most astrometric routines in use generate photometry automatically thus theoretically the increase in astrometric data has led to a corresponding increase in photometric coverage of comet light curves. Unfortunately though, lack of standardisation of amateur CCD data makes its use difficult. CCD magnitudes reported in Minor Planet Electronic Circulars may show a dispersion of 2 or more magnitudes due to the fact that different observers use different apertures, passbands and reference stars for data reduction. In particular, most amateur observers do not use standard filters for their observations and work in "white" light, usually in non-photometric conditions.

In an attempt to standardise CCD photometry of comets and increase its usefulness, Spanish and Italian astrometrists have increasingly adopted a standard method to observe and to reduce data. The basic method is described by Kidger (2002) and involves using a constant 10 arcsec aperture and reducing data against USNO A2.0 " $R$ " stars. Observers are also encouraged to use the same reduction package (most observers use the package Astrometrica ${ }^{1}$ due to its ease of use and robust method of calculating photometry against all stars in the field of view, thus reducing errors).

More recently the method has been extended to include photometry in apertures of 20,30, 40 and 60 arcsec. This allows the coma profile to be measured and thus photometry to be calculated for any aperture, offering the option of calculating photometry for a constant physical aperture of any diameter in kilometres. This option is important to allow the gas and dust production to be measured in a fixed aperture over time. As it is not, at present, a straightforward task to take such multi-aperture photometry with existing packages, a customised routine (FASE3) was developed by Castellano (private communication) to take multi-aperture photometry that includes an improved routine for sky subtraction, using the median of the full field of view rather than the more traditional annulus that will, in extended comets, lead to coma entering in the "sky" aperture. This routine has become widely used by Spanish observers ${ }^{2}$.

For most amateur CCDs the assumption that an unfiltered observation approximates to $R$ is a good one. The calibration of USNO A2.0 photometry against the standard Kron-Cousins $R$ has been tested by Kidger \& Martín-Luis (2003). The USNO " $R$ " photometry is within 0.2 mag of Landolt photometry in $R$ for the range $12<R<19$, which covers the range of magnitudes usually covered by amateur CCD photometry and also

\footnotetext{
1 The Astrometrica package is described at the URL http: //www . astrometrica.at/

2 Modifications to the Astrometrica package are under way that will adapt it to multiaperture photometry and frame median sky determination.
}

the totality of the observations reported here. Note that routines such as Astrometrica and FASE3 measure all the stars in the field of view and discard outliers in the photometric reduction. Although USNO A2.0 is not a photometric catalogue its zeropoint is linked to Tycho stars and the median error in USNO magnitudes in the $R$ band after applying the colour transformation in Eq. (1), is $0.197 \mathrm{mag}$. As this error is comparable with the accuracy that can be obtained for comet photometry with typical amateur telescopes in the $20-30 \mathrm{~cm}$ range we may consider that USNO A2.0 photometry is good enough for our purposes.

We can transform the $R$ magnitude given by USNO A2.0 to the standard Landolt $R$ using the formula

$R_{\text {Landolt }}=0.949 *\left(R_{\mathrm{USNO}}-0.74\right)$.

Applying the above observational techniques to comets we find that particularly in smaller apertures where the sky subtraction is less critical, consistent photometry can be obtained between many observers, with a dispersion in the combined light curve of $0.20 \mathrm{mag}$.

A total of 625 measures of $67 \mathrm{P} /$ Churyumov-Gerasimenko are reported here, although observations are still continuing. These data were obtained by 12 observers on 51 nights. This corresponds to 221 individual integrations of which 101 correspond to multi-aperture photometry and the rest to photometry in the 10 arcsec aperture only. The photometry reported here covers a period from $T-4.4 \mathrm{~d}$ to $T+258 \mathrm{~d}$. The observers and number of points reported by each are shown in Table 1 .

\section{The light curve}

The raw light curve is shown in Fig. 1 after transformation to the Landolt-Kron-Cousins $R$. The $x$-axis is shown as days from perihelion. We see that there is a significant brightening in the 10 arcsec aperture, which has the best-covered data, between $T-4.4$ days and $T+34.7$ days due to a perihelic outburst of amplitude $\approx 1.5 \mathrm{mag}$. This outburst is also shown in other light curves available on the Internet (e.g. the light curve compiled by Seichii Yoshida, http://www.aerith.net/ comet/catalog/0067P/2002.html), and in these is seen to initiate between 0 and 5 days before perihelion. An almost identical outburst was registered at $T-5$ days at the 1996 return (see http://www.aerith.net/comet/ catalog/0067P/1996.html). Marsden (1983) also notes that $67 \mathrm{P} / \mathrm{Churyumov}-G e r a s i m e n k o$ was unusually bright postperihelion during its 1982-83 apparition. Observations made in late 1982 suggest that the comet suffered a significant postperihelion outburst Marsden (1986). Osip et al. (1992) find that Af $\rho$ and the production rate of key species such as $\mathrm{CN}$ and $\mathrm{C}_{2}$ was strongly assymetric about perihelion, consistent with a perihelic outburst similar to those of 1996 and 2002. Morris (1986) shows that maximum of the visual light curve, as determined from total visual magnitude estimates occurred at $T+35$ days. Churyumov \& Filonenko (1991, 1989a,b, 1991) also studied the light curve of this apparition suggest that there were as many as 16 outbursts in all over 6 months of observations and find a possible correlation with variation of the total area of sunspots during the apparition. However, poor coverage of the 
Table 1. Details of observers and observations.

\begin{tabular}{lccccc}
\hline \hline Observer & Telescope & MPC Site Code & Location & No. Points & No. Multiaperture \\
\hline I. Almendros & $0.25-\mathrm{m} \mathrm{S} / \mathrm{C}$ & 212 & Malaga (Spain) & 2 & 0 \\
R. Naves \& M. Campàs & $0.30-\mathrm{m} \mathrm{S} / \mathrm{C}$ & 213 & Catalonia (Spain) & 54 & 25 \\
E. Reina & $0.25-\mathrm{m} \mathrm{S} / \mathrm{C}$ & 232 & Catalonia (Spain) & 66 & 33 \\
R. Ligustri & $0.35-\mathrm{m} \mathrm{S} / \mathrm{C}$ & 235 & Talmassons (Italy) & 11 & 4 \\
A. Sánchez & $0.30-\mathrm{m} \mathrm{S} / \mathrm{C}$ & 442 & Catalonia (Spain) & 3 & 0 \\
M. Camarasa & $0.20-\mathrm{m} \mathrm{S} / \mathrm{C}$ & 445 & Valencia (Spain) & 10 & 3 \\
D. Rodrí́uez & $0.20-\mathrm{m} \mathrm{S/C}$ & 458 & Madrid (Spain) & 2 & 1 \\
J. Castellano & $0.20-\mathrm{m} \mathrm{S} / \mathrm{C}$ & 939 & Catalonia (Spain) & 34 & 18 \\
F. Baldriś & $0.20-\mathrm{m} \mathrm{Newton}$ & A01 & Catalonia (Spain) & 7 & 4 \\
J. Lluís Salto & $0.25-\mathrm{m} \mathrm{S/C}$ & A02 & Catalonia (Spain) & 24 & 7 \\
J. Lacruz & $0.30-\mathrm{m} \mathrm{S/C}$ & J87 & Madrid (Spain) & 5 & 5 \\
C. Pineda & $0.25-\mathrm{m} \mathrm{S/C}$ & J91 & Catalonia (Spain) & 2 & 1 \\
\hline
\end{tabular}

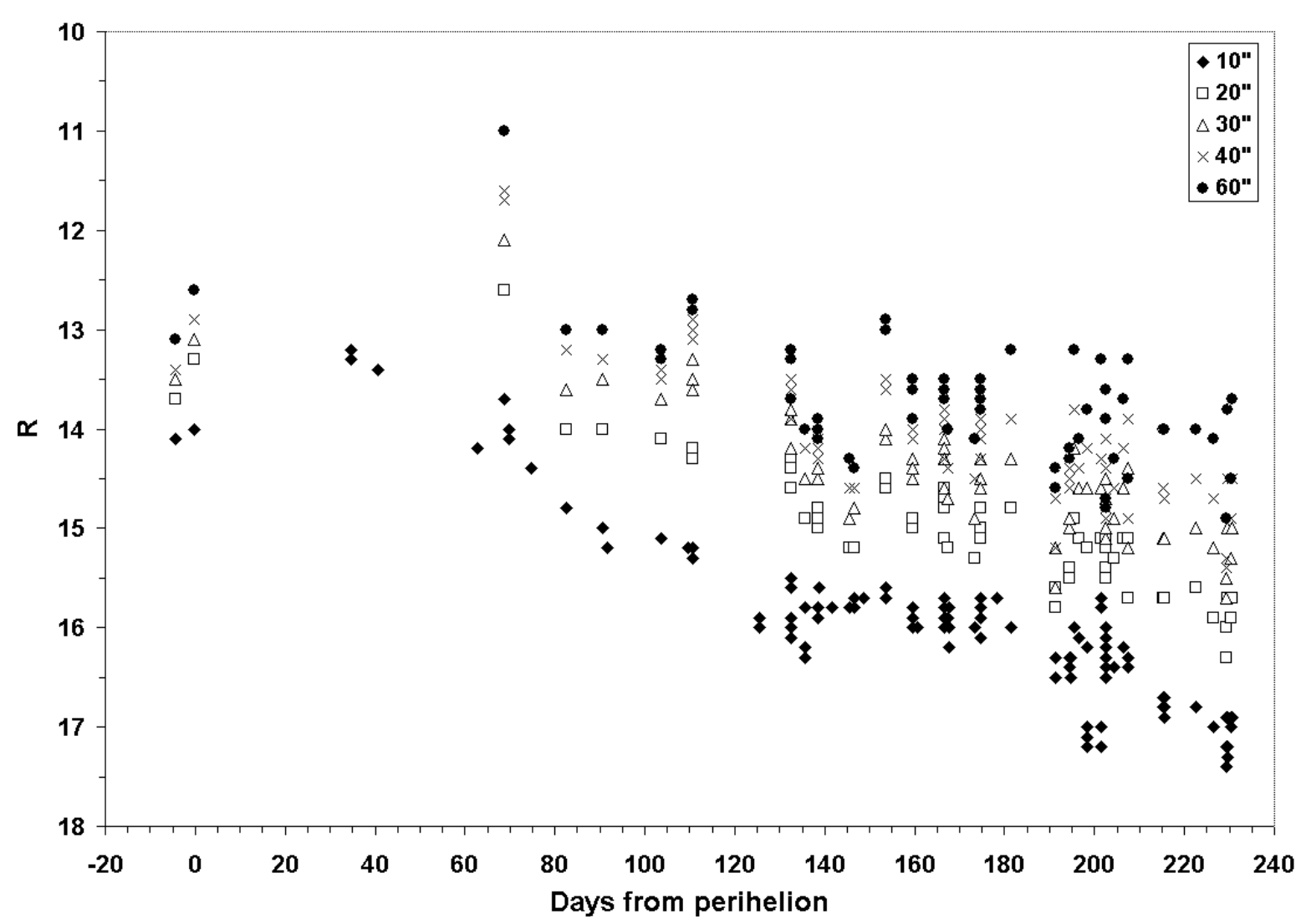

Fig. 1. The raw light curve of 67P/Churyumov-Gerasimenko in $R$ in fixed apertures of 10, 20, 30, 40 and 60 arcsec.

1969-70 and 1975-76 apparitions means that no statement can be made about photometric behaviour prior to 1982 .

Thus perihelic outbursts appear to be a characteristic of the light curve of the comet having been observed at three of the last four returns. It is possible to speculate that a similar outburst will occur at perihelion in 2009. If the outburst sequence continues during the ROSETTA encounter it would offer exciting possibilities for science during a hypothetical extended mission, although it may also invoke significant spacecraft safety issues.
It is possible that the small increase in brightness observed in all apertures between $T-4.4$ days and $T-0.3$ days was due to the initiation of the outburst, although this suggestion has low confidence due to the poor sampling of the light curve. The light curve presented by Yoshida at http://www.aerith. net/comet/catalog/0067P/2002 html is also poorly sampled at this time, thus we can only state that the outburst initiated between $T-5$ days and perihelion and had a fast rise time, reaching maximum in 5 days or less. Note that at $T-4.4$ days the physical radius of the 10 arcsec aperture is $6300 \mathrm{~km}$. For a 


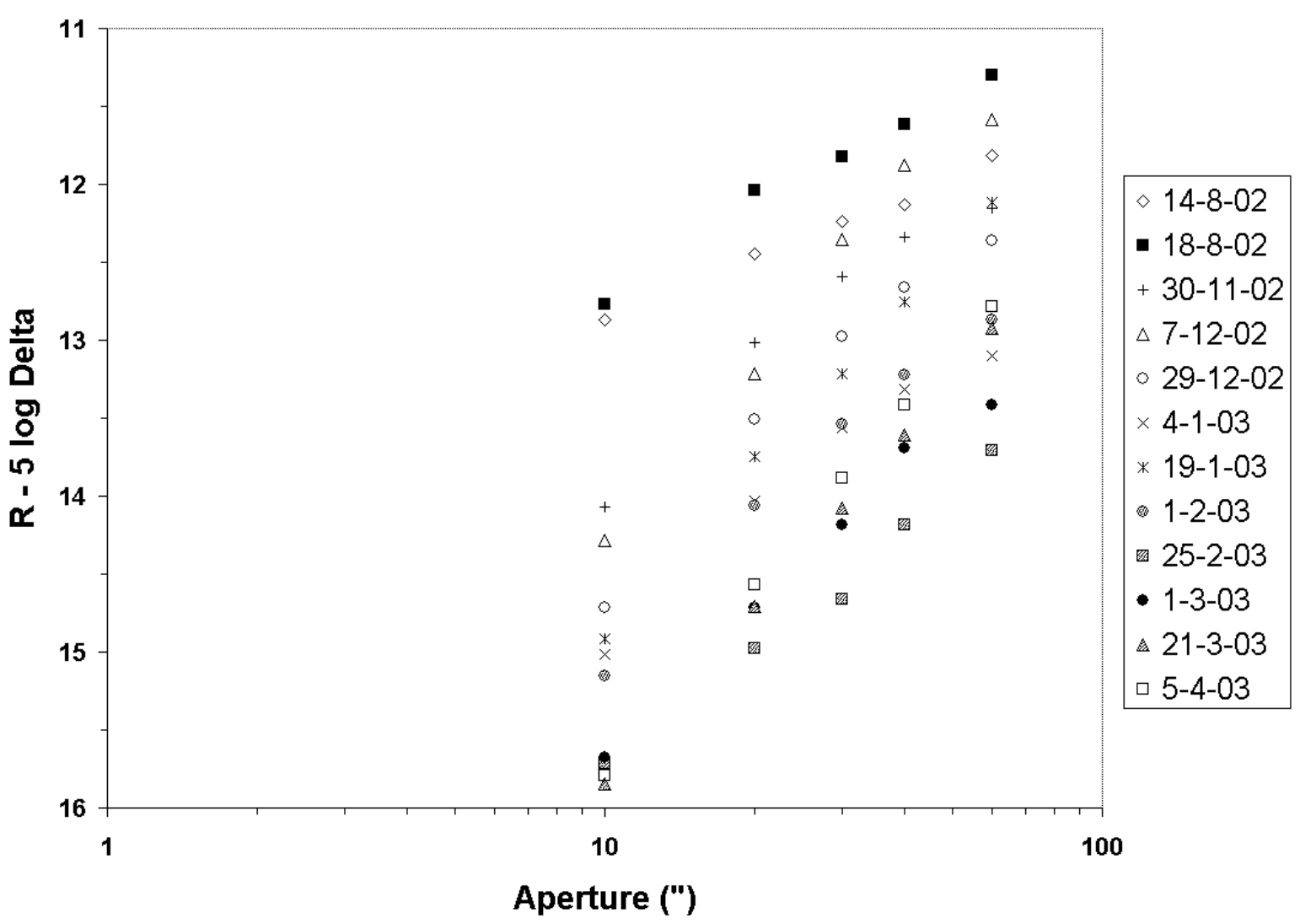

Fig. 2. Coma profiles for 67P/Churyumov-Gerasimenko in $R$ from a representative sample of photometry in apertures of $10,20,30,40$ and 60 arcsec. The photometry is corrected for the geocentric distance. The least squares fits to the photometry are not shown to reduce confusion in the plot.

dust emission event with an expansion velocity of $30 \mathrm{~m} \mathrm{~s}^{-1}$ the crossing time for even the 10 arcsec aperture is $58 \mathrm{~h}$. The rate of rise of the light curve both in 1996 and 2002 suggests that the rise time for the total visual magnitude of the integrated coma is faster than this and thus that the outburst is caused principally by gas release. A second, smaller and slower outburst event is seen at $\approx T+130$ days.

The archive of multiaperture photometry allows us to obtain basic information about the coma morphology during the 2002-2003 apparition. We may approximate the coma profile by fitting the photometry in each aperture for each individual date of the type

$R=\log a+b \log r$.

Where: " $R$ " is the magnitude in $R$; " $r$ " is the diameter of the aperture in arcseconds; and " $a$ " and " $b$ " are constants. The constant " $b$ " we refer to as the coma index, while " $a$ " is related in a non-trivial fashion to the absolute magnitude, but will not be further considered here.

Coma profiles for selected dates are shown in Fig. 2. The profile which is shown is the mean of all observations reported for that date. For a "typical" comet which has a $1 / r$ brightness distribution we find a slope of the coma of $b \approx-2.5$.

The profiles show a well defined steepening with time from perihelion. In these plots a flatter profile implies a more condensed coma with a greater concentration of light in the nucleus. This corresponds with the observed morphological evolution in the corresponding CCD images these are available in chronological order at http://www.iac.es/galeria/ $\mathrm{mrk} /$ comets $/ 67 \mathrm{p} / 67 \mathrm{p} . \mathrm{htm}$ that shows a progressive change from a strongly centrally condensed coma close to perihelion to a very weakly condensed, difuse coma at $T+258$ days. The variation of the coma index with time is shown in Fig. 3.

Although using an aperture of a fixed angular diameter gives information on light curve structure and behaviour, it has the disadvantage of measuring a variable quantity of light from the coma as a function of heliocentric and geocentric distance. To measure the gas and dust production from the nucleus as a function of the heliocentric distance it is necessary to measure in an aperture of a fixed physical diameter. This can be done by using the multiaperture photometry to calculate the photometry for any given aperture.

To measure the near-nucleus activity we define a physical aperture of $10000 \mathrm{~km}$. This is close to the equivalent size of the 10 arcsec aperture at the typical geocentric distance during the 2002-2003 apparition of 67P/Churyumov-Gerasimenko. This aperture is choosen because inspection of the light curve shows that it has the smallest dispersion as it is least sensitive to errors in measuring the exact level of the sky background around the comet. The photometry in this aperture is is combined with the measured coma index to calculate the equivalent magnitude in the $10000 \mathrm{~km}$ diameter aperture at a given epoch. In an attempt to reduce both the dispersion and the intrinsic errors propagated in the conversion we use only the measurement in the $10 \operatorname{arcsec}$ 


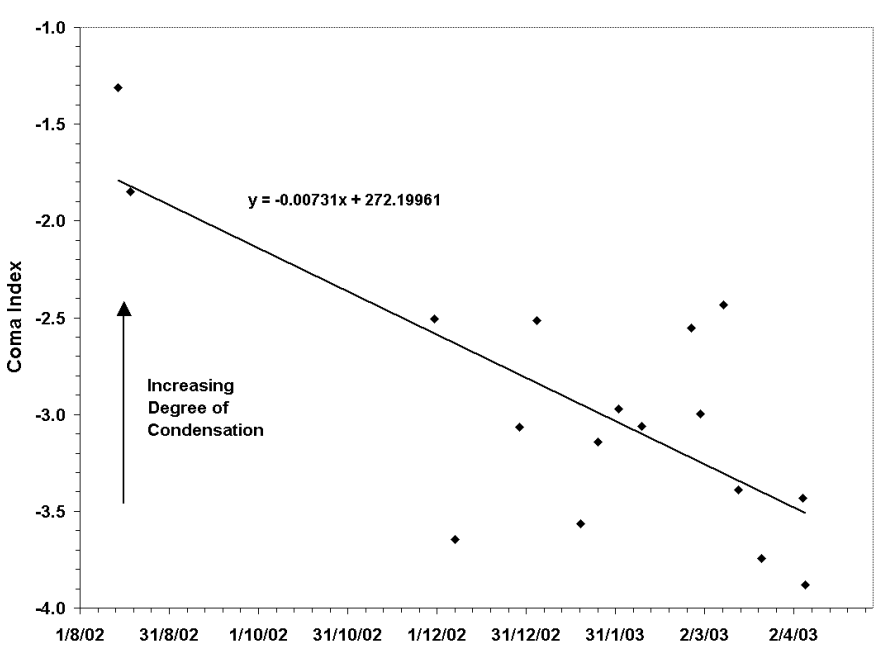

Fig. 3. Variation of the coma index of $67 \mathrm{P} /$ Churyumov-Gerasimenko with time. The variation can be modelled as a simple least squares regression against time.

aperture combined with the coma index for a given date calculated from Fig. 3 to calculate the equivalent magnitude in the $10000 \mathrm{~km}$ aperture.

The resultant plot is shown in Fig. 4. The $R$ magnitude is shown, corrected for geocentric distance, for a fixed aperture of $10000 \mathrm{~km}$.

One of the most useful measures of cometary activity is to calculate the variation in the magnitude corrected for geocentric distance against the logarithm of heliocentric distance. This is shown in Fig. 5. Three distinct regimes are seen. There is a rather poorly covered perihelic outburst of approximately 1 mag amplitude in this $10000 \mathrm{~km}$ aperture. This is followed by a monotonic fade to a distance of $r=1.9$ AU. At $1.9 \mathrm{AU}$ a slow outburst of approximately $0.5 \mathrm{mag}$ amplitude occurs. The slow rate of rise to maximum indicates that the aperture crossing time was long and thus that this was probably a dust-generated event produced by an injection of rather large, slow-moving grains.

If we ignore the rise to maximum at perihelion, which is too poorly covered to study in detail, the light curve can be considered as three individual segments:

$$
\begin{aligned}
& -r<1.91 \mathrm{AU} \\
& R(10000 \mathrm{~km})=10.15+5 \log \Delta+18.05 \log r \\
& -1.91<r<2.27 \mathrm{AU} \\
& R(10000 \mathrm{~km})=15.18+5 \log \Delta+0.22 \log r \\
& -r>2.27 \mathrm{AU} \\
& R(10000 \mathrm{~km})=5.38+5 \log \Delta+26.95 \log r .
\end{aligned}
$$

Where " $R(10000 \mathrm{~km})$ " is the $R$ magnitude integrated over a 10 arcsec aperture using the transformation defined in Eq. (1) to convert the raw data to the standard Johnson-Kron-Cousins scale.

Note that after the late outburst the rate of fade of brightness increases significantly, consistent with a sharply decreased gas and dust emission rate.

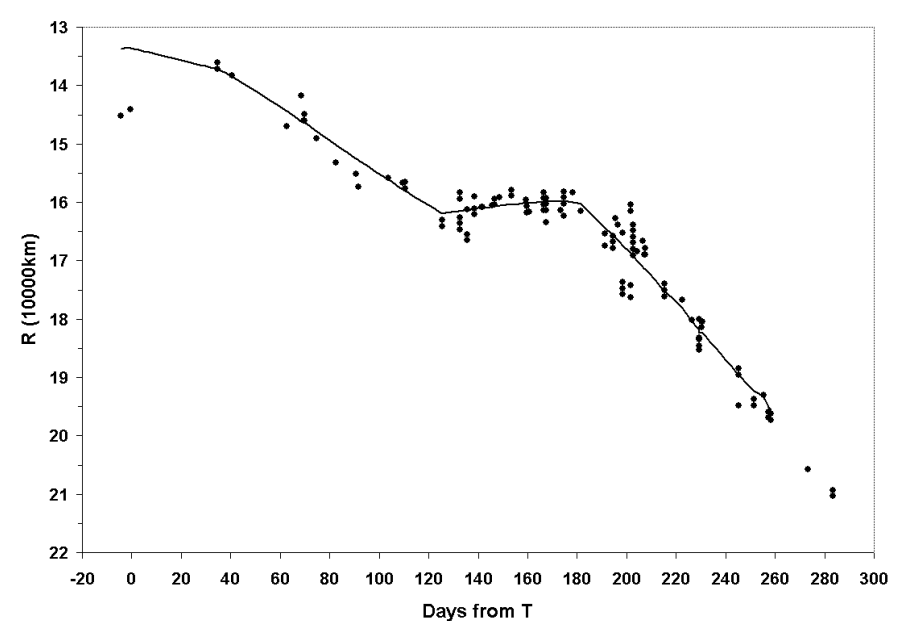

Fig. 4. The light curve of 67P/Churyumov-Gerasimenko in $R$ in an aperture of $10000 \mathrm{~km}$ plotted against time from perihelion. The light curve fit from Eqs. (2)-(4) is superimposed and extrapolated back to perihelion to show the amplitude of the outburst in this aperture.

A useful parameter for discussing the rate of activity of the nucleus is $A f \rho$. This measures the product of the albedo of dust and filling factor for an aperture $\rho$.

Af $\rho$ in centimetres can be calculated from the relation

$A F \rho=\mathrm{e}^{\left.(2 \ln (\Delta+r)-\ln \delta+\ln \rho)-\left(M_{\odot}+m\right) / 1.086+50.546\right)}$.

Where

- $\Delta$ and $r$ are the geocentric and heliocentric distances in AU; - $\delta$ is the geocentric distance in kilometres;

- $\rho$ is the diameter of the photometric aperture in kilometres;

- $M_{\odot}$ is the solar absolute magnitude in the observed band;

- $m$ is the observed magnitude of the comet in aperture $\rho$.

To calculate $A f \rho$ we use the data for the constant physical aperture of $10000 \mathrm{~km}$ derived in Fig. 3. The variation of $A f \rho$ with time is shown in Fig. 6. Errors of 0.20 mag are assumed in the magnitudes used to calculate the values of $A f \rho$. We see that the value measured in $R$ increases from $135 \mathrm{~cm}$ at perihelion, to $290 \mathrm{~cm}$ at $T+35$ days. Unfortunately no photometry is available at the peak of the outburst, but it can be assumed from the extrapolation of the decline in values that the maximum value of $A f \rho$ was $\approx 350 \mathrm{~cm}$, thus the outburst at perihelion increased the dust production as measured by $A f \rho$ by a factor of close to 3 .

The value of $A f \rho$ dropped rapidly from the peak of the outburst as the heliocentric distance increased. At $T+90$ days it had decreased to $\approx 60 \mathrm{~cm}$, a decrease of a factor of 6 from maximum, although the heliocentric distance increased only from $r=1.29$ to $r=1.67$ AU. Only a small increase in Af $\rho$ is seen at the time of the late outburst. The typical values measured increased from $40-50 \mathrm{~cm}$ pre-outburst to $50-60 \mathrm{~cm}$ at maximum however, when we plot $\log A f \rho$ against time we see that the outburst was a substantial one over the extrapolation of the decline from $T+35$ days to $T+125$ days. The extrapolated value of $A f \rho$ at $T+170$ days was $\sim 10 \mathrm{~cm}$, but the measured value at this epoch was $\sim 50 \mathrm{~cm}$, a factor of 5 higher than expected at this epoch, hence $67 \mathrm{P} / \mathrm{Churyumov}-G e r a s i m e n k o$ is capable of 


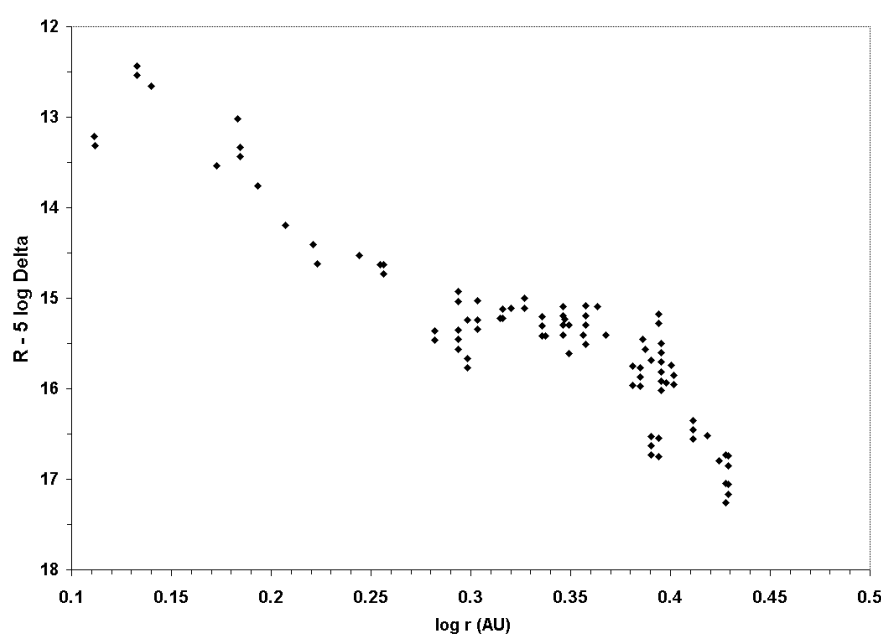

Fig. 5. The light curve of 67P/Churyumov-Gerasimenko in $R$ in an aperture of $10000 \mathrm{~km}$, corrected for geocentric distance, plotted against the logarithm of the heliocentric distance.

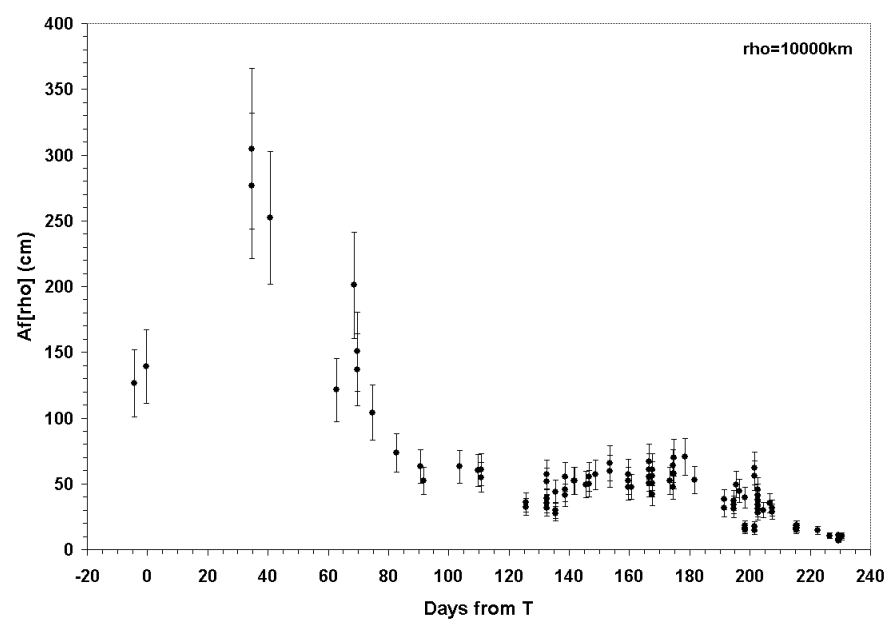

Fig. 6. Variation of $A f \rho$ for $67 \mathrm{P} /$ Churyumov-Gerasimenko with time in $R$ in a $10000 \mathrm{~km}$ aperture.

significant outburst activity even at $r=2.2$ AU post-perihelion. This outburst shows a flat maximum over $\approx 60$ days before declining rapidly from $T+200$ days.

Af $\rho$ was also calculated for the aperture of $60000 \mathrm{~km}$ (Fig. 7), corresponding closely to the aperture of $60 \mathrm{arcsec}$. Note that the sampling in the 60 arcsec aperture is significantly poorer particularly before $T+100$ days and shows larger scatter due to the difficulty in obtaining an accurate sky subtraction with a large aperture and faint, extended coma. The $60000 \mathrm{~km}$ aperture takes in the majority of the coma of the comet. The magnitude in this aperture is more susceptible to sky subtraction errors, particularly in the relatively light-polluted sites used by many of the observers, where a small error in the determination of the sky background level may cause significant errors in the photometry of the coma in a large aperture, hence the dispersion in the data is much larger than for the smaller aperture. However, we see that the pattern of activity is completely different. The data shows a constant value of $A f \rho \approx 80 \mathrm{~cm}$ apart from a value of $460 \mathrm{~cm}$ at $T+69$ days. This high value is registered during the outburst and may be genuine as it is the

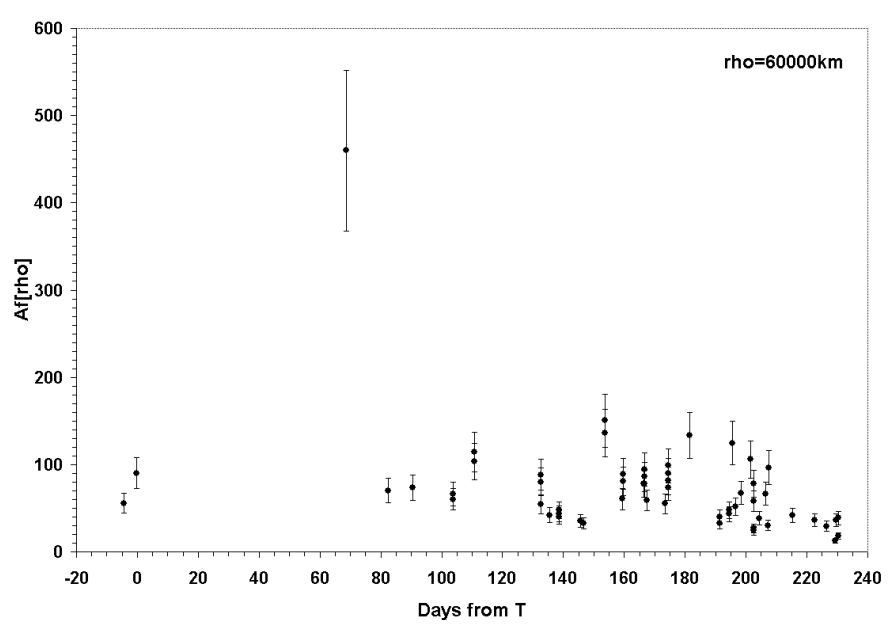

Fig. 7. Variation of $A f \rho$ for $67 \mathrm{P} /$ Churyumov-Gerasimenko with time in $R$ in a $60000 \mathrm{~km}$ aperture.

mean of 4 individual measures taken on that night, although the same observer registers values of $70 \mathrm{~cm}$ and $73 \mathrm{~cm}$ respectively at $T+83$ and $T+91$ days respectively. Even if this value is accepted, the lack of time variation of $A f \rho$ in the $60000 \mathrm{~km}$ aperture is surprising. Only at $T>210$-days is a clear variation seen, with a rapid decline observed in the $A f \rho$ values from the plateau observed from $T-4$ days on. Note that the data for apertures of intermediate sizes of 20,30 and 40 arcsec show a light curve behaviour that tends to that of the 60 arcsec aperture as the aperture size increases.

\section{Discussion}

Combining the estimated radius of the nucleus of Tancredi et al. (2000) with the production rates measured by Osip et al. (1992) suggests that the active area of the nucleus of 67P/ChuryumovGerasimenko is $\approx 6 \%$. The slightly lower radii estimated by Kamoun et al. (1998) and Mueller (1992) would lead to a corresponding increase in active area.

The presence of major outburst activity though may cause this fraction to change significantly even during a single apparition. The large rise in $A f \rho$ seen around perihelion is similar to that seen by Osip et al. (1992) during the 1982 apparition. At that time sharp rises were seen around perihelion in the $\mathrm{OH}$ and $\mathrm{CN}$ production rate, as well as in $A f \rho$. Unfortunately the sampling of their data is poor and it is not possible to state when the outburst initiated. A peak value of $A f \rho=450 \mathrm{~cm}$ was recorded, similar to the peak values reported here. Strong similarities in the light curve are seen in the 1982-83, 1996-97 and 2002-2003 apparitions suggesting that the light curve is repetitative over several returns. This has important implications for the ROSETTA mission, particularly if the perihelion outbursts seen at these three apparitions repeat at the 2009 apparition and, even more importantly during the ROSETTA encounter. However, even the peak values of $A f \rho$ at outburst $(\approx 450 \mathrm{~cm})$ are still a factor of $\sim 40$ lower than the values of 20 000-30000 cm measured for 1P/Halley in 1986.

The values of $A f \rho$ found here are consistent with the gas and dust production rates calculated by Hanner et al. (1985) 


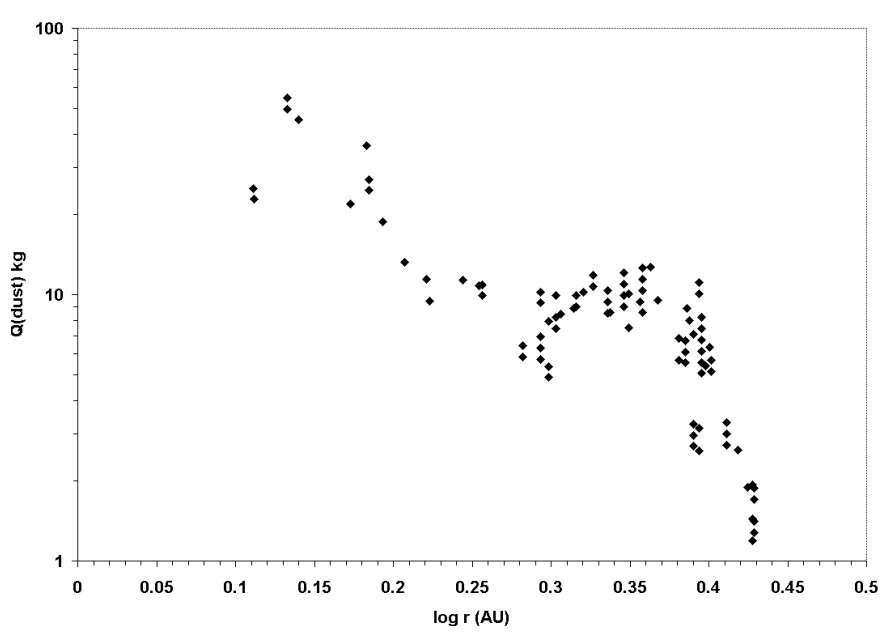

Fig. 8. The dust production rate $Q_{\mathrm{d}}$ for $67 \mathrm{P} /$ Churyumov-Gerasimenko during the 2002-2003 apparition, estimated from the data presented in the text assuming that the dust characteristics are similar to those of the 1982-83 return. Two major outbursts are seen.

during the 1982-83 apparition. However, while Af $\rho$ is in principal aperture insensitive, the measured dust production rate $\left(Q_{\mathrm{d}}\right)$ is strongly model dependent, relying on assumptions about the density, and the size and velocity distribution of grains. Of these parameters, the density is the poorest known and most authors use a canonical value of $1 \mathrm{~g} / \mathrm{cm}^{3}$. Model uncertainties can lead to a factor of $\sim 2$ difference between different determinations of $Q_{\mathrm{d}}$.

Krishna Swami (1991) finds large variations in $Q_{\mathrm{d}}$ from $17-74 \mathrm{~kg} \mathrm{~s}^{-1}$ recalculating the data presented by Hanner et al. (1985), who calculate much larger values $\approx 110 \mathrm{~kg} \mathrm{~s}^{-1}$, with a peak value at $r=1.37 \mathrm{AU}$ of $Q_{\mathrm{d}}=220 \mathrm{~kg} \mathrm{~s}^{-1}$. The reasons for these differences are due to two factors: (i) a slightly different form of the grain size distribution; and (ii) the assumption of mostly absorbing grains.

Osip et al. (1992) find values of $Q_{\mathrm{H}_{2} \mathrm{O}}=110 \mathrm{~kg} \mathrm{~s}^{-1}$ and $Q_{\mathrm{d}}=54 \mathrm{~kg} \mathrm{~s}^{-1}$ at $r=1.5 \mathrm{AU}$, with an implied gas to dust ratio of 2 . This classes $67 \mathrm{P} / \mathrm{Churyumov-Gerasimenko} \mathrm{as} \mathrm{a} \mathrm{dusty}$ comet (Krishna Swami 1991). These values of $Q_{\mathrm{H}_{2} \mathrm{O}}$ are also a factor of $\approx 40$ lower than the corresponding ones for $1 \mathrm{P} /$ Halley in 1986. Another production rate from radio $\mathrm{OH}$ at Nancay was published by Crovisier et al. (2002): $Q[\mathrm{OH}]=0.9 \times 10^{28} \mathrm{~s}^{-1}$ for mid-October 1982, equivalent to $270 \mathrm{~kg} \mathrm{~s}^{-1}$. The difference with Osip et al. (1992) could be attributed to the different authors using distinct Haser-model assumptions.

The value of $A f \rho=300 \mathrm{~cm}$ at $r=1.5$ AU measured by Osip et el. (1992) in corresponds to a calculated value of $Q_{\mathrm{d}}=54 \mathrm{~kg} \mathrm{~s}^{-1}$. Assuming that the characteristics of the dust emitted from the nucleus have been similiar in 2002-2003 to those of 1982-83, the peak dust production during the outburst at perihelion was $\approx 65 \mathrm{~kg} \mathrm{~s}^{-1}$, but falls to $10 \mathrm{~kg} \mathrm{~s}^{-1}$ at $T+110$ days, equivalent to $r=1.60 \mathrm{AU}$ (see Fig. 8). Given these assumptions, we can calculate that dust production thus falls as $r^{-5.8}$ during the 2002-2003 apparition, similar to the production rate of $\mathrm{OH}$ in 1982-83 ( $r^{-5.8}$, Osip et al. 1992), and much steeper than the measured dust production rate at that apparition $\left(r^{-3}\right)$. However, $Q_{\mathrm{d}}$ rose from $7 \mathrm{~kg} \mathrm{~s}^{-1}$ to $>10 \mathrm{~kg} \mathrm{~s}^{-1}$ between $r=1.9 \mathrm{AU}$ and $r=2.2 \mathrm{AU}$, before returning to its previous $r^{-5.8}$ dependence. Thus the value of the " $r$ " dependence measured by Osip et al. (1992) in 1982-83 would be sensitive to possible similar outbursts that would flatten the observed dependence over a limited range of heliocentric distance. If we take only the data for the 2002-2003 apparition from perihelion to the peak of the second outburst, the apparent dependence flattens greatly and a value close to the $r^{-3}$ index of 1982-83 would be obtained.

\section{Conclusions}

It has been shown that carefully treated and normalised amateur CCD data of comets is a potentially extremely valuable resource for the study of cometary light curves. The data taken in 2002-2003 shows a perihelic outburst very similar to those seen in 1982-83 and in 1996-97. This suggests that the light curve is consistent over several returns to perihelion allowing the potential for a high level of predictability of activity for a ROSETTA encounter in 2014, although it is true that the characterisation and landing on the nucleus will take place during the pre-perihelion phase that is regretably not covered by these data, and has historically been little covered in the light curve at any previous return.

Of particular interest in the light curve is the apparent reproducibility of perihelic outbursts over various returns that lead to an increase of a factor of $\sim 3$ in dust production. Such an outburst during the 2015 return would provide an exciting science opportunity for a ROSETTA mission extended through perihelion. Such close-up observations of an outburst could invoke spacecraft safety issues, although even in outburst it must be stressed that $67 \mathrm{P} /$ Churyumov-Gerasimenko is more than an order of magnitude less active than $1 \mathrm{P} /$ Halley.

Acknowledgements. This paper would not have been possible without the help and support of the observers of the Observadores_cometas mailing list http://groups. yahoo.com/ group/Observadores_cometas/. In particular R. Naves and M. Campàs have been highly supportive of this project. The multiaperture data presented here has been reduced using the FASE3 routine developed by J. Castellano for the observers of this list. The development of the observing method described has been aided by several valuable conversations with B. Marsden at the Central Bureau for Astronomical Telegrams. The author is pleased to acknowledge a series of helpful and valuable comments from J. Crovisier that have significantly improved this manuscript.

\section{References}

Beliaev, N. A. 1974, in Asteroids, comets, meteoric matter (A75-26778 11-89) (Bucharest: Editura Academiei Republicii Socialiste Romania), 149

Beliaev, N. A., Kresak, L., \& Pittich, E. M. 1986, in Catalogue of short-period comets (Bratislava: Slovak Academy of Sciences, Astronomical Institute)

Carusi, A., Kresak, L., Perozzi, E., \& Valsecchi, G. B. 1985, in Long-term evolution of short-period comets Bristol (England and Accord, MA, Adam Hilger, Ltd.), 272 
Churyumov, K. I., \& Filonenko, V. S. 1989a, Kiev Comet Circ., 403,5

Churyumov, K. I., \& Filonenko, V. S. 1989b, Kiev Comet Circ., 404,3

Churyumov, K. I., \& Filonenko, V. S. 1991, Sov. Astron. Lett., 417,470

Churyumov, K. I., \& Filonenko, V. S. 1992, in Lunar and Planetary Inst., Asteroids, Comets, Meteors 1991, 121

Crovisier, J., Colom, P., Gérard, E., Bockelée-Morvan, D., \& Bourgois, G. 2002, A\&A, 393, 1053

Hanner, M. S., Tedesco, E., Tokunaga, A. T., et al. 1985, Icarus, 64, 11

Kamoun, P., Campbell, D., Pettengill, G., \& Shapiro, I. 1998, P\&SS, 47,23
Kidger, M. R. 2002, EM\&P, 90, 259

Kidger, M. R., \& Martín-Luis, F. 2003, in preparation

Kiselev, N. N., \& Velichko, F. P. 1998, Icarus, 133, 286

Krishna Swami, K. S. 1991, A\&A, 241, 260

Marsden, B. G. 1983, IAUC, 3764

Marsden, B. G. 1986, QJRAS, 27, 102

Morris, C. 1986, Icarus, 64, 11

Mueller, B. E. A. 1992, in Lunar and Planetary Inst., Asteroids, Comets, Meteors 1991, 425

Osip, D., Schleicher, D. G., \& Millis, R. L. 1992, Icarus, 98, 115

Tancredi, G., Fernández, J. A., Rickman, H., \& Licandro, J. 2000, A\&AS, 146, 73 